\title{
Using Cloud Technology to Support Monitoring During High Profile Events
}

\author{
Megan Patel ${ }^{\star 1}$, Enyinnaya Adighibe ${ }^{1}$, Joseph Lombardo ${ }^{2}$, Wayne Loschen ${ }^{2}$, Miles Stewart ${ }^{2}$ \\ and Michael O. Vernon ${ }^{1}$ \\ ${ }^{1}$ Cook County Department of Public Health, Oak Forest, IL, USA; 2Johns Hopkins University Applied Physics Laboratory, Laurel, MD, \\ USA
}

\section{Objective}

In May 2012, thousands of protesters, descended on Chicago during the NATO Summit to voice their concern about social and economic inequality. Given the increased numbers of international and domestic visitors to the Windy City and the tension surrounding protesting during the summit, increased monitoring for health events within the city and Chicago metropolitan region was advised. This project represents the first use of cloud technology to support monitoring for a high profile event.

\section{Introduction}

Hospital emergency departments in Cook and surrounding counties currently send data to the Cook County Department of Public Health $(\mathrm{CCDPH})$ instance of ESSENCE on CCDPH servers. The cloud instance of ESSENCE has been enhanced to receive and export all meaningful use data elements in the meaningful use format. The NATO summit provided the opportunity for a demonstration project to assess the ability of an Amazon GovCloud instance of ESSENCE to ingest and process meaningful use data, and to export meaningful use surveillance data to the Cook County Locker in BioSense 2.0

\section{Methods}

In the three weeks leading up to the NATO Summit, HL7 data extracts were sent to BioSense 2.0 and a data feed was established to the Amazon GovCloud instance of ESSENCE. Queries specific to anticipated health events associated with the summit such as injuries, tear gas exposure, and general exposure, were developed. Several features of the cloud instance of ESSENCE enhanced the ability of CCDPH staff epidemiologists to conduct analyses, including the sharing capabilities of queries and the myESSENCE dashboard feature. The sharing capabilities within the cloud instance of ESSENCE allowed queries to be easily shared with multiple staff epidemiologists and across health jurisdictions. The myESSENCE dashboard feature was used to create dashboards of surveillance results, including time series graphs, maps, and records of interest for relevant queries, that were shared with public health staff monitoring population health during the summit. This information was used to provide situational awareness on a daily basis in the Chicago Metropolitan region.

\section{Results}

Data feeds to BioSense 2.0 and the Amazon GovCloud instance of ESSENCE were successful. The NATO Summit did not produce any remarkable public health concerns in suburban Cook County. The use of the cloud instance of ESSENCE enhanced the timeliness of generating situational awareness reports for distribution to public health partners in the Chicago Metropolitan region.

\section{Conclusions}

While further evaluation of cloud resources to conduct syndromic surveillance is warranted, use of the cloud instance of ESSENCE during the NATO Summit demonstrated the ability of the cloud to support surveillance for both routine and high profile events.

\section{Keywords}

Cloud; Meaningful Use; Surveillance; Public Health

\section{Acknowledgments}

This project was supported by the Wide Area Recovery and Resiliency Program of the Department of Homeland Security under contract N00024-03-6606. Cloud services were provided by the CDC's BioSense 2.0 Program under contract 200-2011-40934.

\section{*Megan Pate}

E-mail: mctoth@gmail.com 